\title{
Parallel Chip Firing Game Associated with n-cube Edges Orientations
}

\author{
René Ndoundam ${ }^{1}$, Claude Tadonki ${ }^{2}$, and Maurice Tchuente ${ }^{1}$ \\ 1 University of Yaoundé I, Faculty of Science, \\ Department of Computer Science, P.O. Box. 812 Yaoundé, Cameroon \\ 2 University of Geneva, Centre Universitaire Informatique, \\ Departement of Theoretical Computer Science 24, rue Général Dufour, \\ 1211 Genève 4 - Switzerland \\ ndoundam@uycdc.uninet.cm, claude.tadonki@cui.unige.ch
}

\begin{abstract}
We study the cycles generated by the chip firing game associated with $n$-cube orientations. We consider a particular class of partitions of vertices of $n$-cubes called left cyclic partitions that induce parallel periodic evolutions. Using this combinatorical model, we show that cycles generated by parallel evolutions are of even lengths from 2 to $2^{n}$ on $H_{n}(n \geq 1)$, and of odd lengths different from 3 and ranging from 1 to $2^{n-1}-1$ on $H_{n}(n \geq 4)$. However, the question weather there exist parallel evolutions with period greater that $2^{n}$ remains opened.
\end{abstract}

\section{Introduction}

A state in the parallel chip firing game played on a directed graph $G=(X, A)$ is a mapping $x: V \rightarrow N$ which can be viewed as a distribution of chips onto the vertices of $G$. In a transition of the game, a state $x$ is transformed into a new state by activating all nodes with more chips that its out-neighbors. The evolution is ultimately periodic because the total number of chips remains constant. More precisely, if $x^{t}, t \geq 0$, denotes the state of the system at time $t$, then there exists an integer $q$ called transient length and another integer $p$ called period or cycle length such that

$$
x^{t+p}=x^{t} \text { for } t \geq q \text {, and } x^{t+p^{\prime}} \neq x^{t} \text { for } p^{\prime}<p .
$$

In this paper, we investigate the dynamics generated by the chip firing game associated with $n$-cube orientations and we provide a model to study possible periods generated in this particular case.

\section{Recurrent Construction of Parallel Cycles}

Definition 1. A partition $S_{0} \cup S_{1} \cup \ldots \cup S_{k-1}$ of the vertices of an $n$-cube is called a left cyclic partition if the two following statements hold.

- For all $i$ from 0 to $k-1$, every vertex of $S_{i}$ has a neighbor in $S_{i-1}$, where index operations are performed modulo $k$.

- For all $i$ from 0 to $k-1$, there is no edge between two vertices of $S_{i}$. 
The model of left cyclic partition clearly gives a characteristic of parallel evolutions with unique firing within a cycle. In this paper, we investigate on possible configurations. We first present the construction of left cyclic partitions of even lengths.

Lemma 1. An n-cube admits left cyclic partitions of all even lengths from 2 to $2^{n}$.

Proof. Let $H_{n}=(V, E)$ be an $n$-cube an let $p$ be an even integer between 2 and $2^{n}$. It is well known that, since $p$ is even, there is a cycle $\left[x_{0}, x_{1}, \ldots, x_{p-1}, x_{0}\right]$ of length $p$ in $H_{n}$. Now, for every vertex $u$, let $\Gamma(u)$ denote the set of all neighbors of $u$ in $H_{n}$. This notation is naturally extended to a set of vertices. A left cyclic partition of order $p$ is obtained as follows.

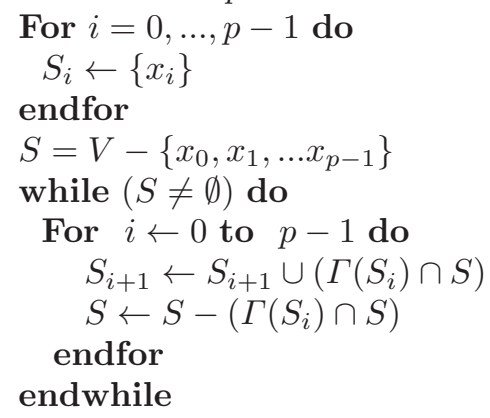

It is obvious that $S_{0}, \ldots, S_{p-1}$ is a partition of $V$ and that every vertex in $S_{i}$ has at least one neighbor in $S_{i-1}$. So we just need to show that two vertices of the same subset $S_{i}$ cannot be neighbors. Let $a$ and $b$ be two vertices of $S_{i}$.

- There is a path from a to $x_{0}$ of length $\ell_{1}$ such that $\ell_{1}=i \bmod p$,

- There is a path from b to $x_{0}$ of length $\ell_{2}$ such that $\ell_{2}=i \bmod p$,

Since $p$ is even, it follows that $\ell_{1}=\ell_{2} \bmod 2$. Hence, if $a$ and $b$ were neighbors, there would exist a cyclic path of odd length $\ell_{1}+\ell_{2}+1$ joining $a$ and $b$ in $H_{n}$, which is not possible since $H_{n}$ is a bipartite graph. This shows that two vertices of the same subset cannot be neighbors.

We now turn to the construction of left cyclic partitions of odd lengths.

Lemma 2. If $S_{0}, S_{1}, S_{2}$ is a left cyclic partition of $H_{n}, n \geq 2$, then every vertex of $S_{i}$ has at least two neighbors in $S_{i-1}$ for $i=0,1,2$.

Proof. Because of symmetry considerations, we can assume that $i=2$. So let $x$ be a vertex of $S_{2}$. From the definition of left cyclic partitions,

- $x$ has a neighbor $x \oplus e_{j}$ in $S_{1}$, where $\oplus$ is the XOR operator and $e_{j}$ is a vector of the canonical basis.

- similarly, $x \oplus e_{j}$ has a neighbor $x \oplus e_{j} \oplus e_{k}$ in $S_{0}$.

Now consider the vertex $x \oplus e_{k}$.

- It is a neighbor of $x$, hence it does not belong to $S_{2}$.

- It is a neighbor of $x \oplus e_{j} \oplus e_{k}$, hence it does not belong to $S_{0}$.

It then follows that $x \oplus e_{k}$ belongs to $S_{1}$, hence $x$ admits two neighbors $x \oplus e_{j}$ and $x \oplus e_{k}$ which are both in $S_{1}$. 
Lemma 3. If $H_{n}, n \geq 3$ admits a left cyclic partition of order 3 , then $H_{n-1}$ admits a left cyclic partition of order 3 .

Proof. Obvious.

Proposition 1. n-cubes do not admit left cyclic partitions of order 3.

Proof. From lemma 2, if a hypercube $H$ admits a left-cyclic partition of order 3 , then $|H| \geq 6$, which is not the case for $H_{2}$. By application of lemma 3, we deduce that no $n$-cube, $n \geq 3$ admits a left cyclic partition of order 3 .

Proposition 2. If $S_{0}, \ldots, S_{p-1}$ is a left cyclic partition of odd order $p$ of $H_{n}$, then $p \leq 2^{n-1}-1$.

Proof. We just have to show that in such a case, $\left|S_{i}\right| \geq 2$ for $i=0, \ldots, p-1$. Indeed, starting from a vertex $a_{p-1} \in S_{p-1}$, we construct a chain $\left[a_{p-1}, a_{p-2}, \ldots\right.$ , $\left.a_{0}, b_{p-1}, b_{p-2}, \ldots, b_{0}\right]$ such that $a_{i}, b_{i} \in S_{i}$ for $i=0, \ldots, p-1$. It is clear that $a_{i} \neq b_{i}, i=0, \ldots, p-1$, otherwise we would have displayed a closed path of odd length in $H_{n}$ which is not possible.

Lemma 4. If $H_{n}$ admits a left cyclic partition of order $p$, then $H_{n+1}$ admits left cyclic partition of order $p$.

Proof. If $S_{0}, \ldots, S_{p-1}$ is a left cyclic partition of order $p$ in $H_{n}$, then $1 S_{i} \cup$ $0 S_{i-1}, i=0, \ldots, p-1$ is a also left cyclic partition of order $p$ in $H_{n+1}$.

Lemma 5. If $H_{n}$ admits a left cyclic partition of odd order $p, p \geq 5$ then $H_{n+1}$ admits a left cyclic partition of order $2 p-1$. Moreover, if $p \geq 7$, then $H_{n+1}$ admits a left cyclic partition of order $2 p-3$.

Proof. Let $S_{0}, S_{1}, \ldots, S_{p-1}$ be a left cyclic partition of odd order $p$.

- Case $p \geq 5$

The following sequence is a left cyclic partition of order $2 p-1$ in $H_{n+1}$.

$0 S_{0}, 1 S_{0} \cup 0 S_{1}, 1 S_{1}, 1 S_{2}, 0 S_{2}, 0 S_{3}, 1 S_{3} \ldots, 1 S_{2 i}, 0 S_{2 i}, 0 S_{2 i+1}, 1 S_{2 i+1}, \ldots, 1 S_{p-3}$, $0 S_{p-3}, 0 S_{p-2}, 1 S_{p-2}, 1 S_{p-1}, 0 S_{p-1}$.

- Case $p \geq 7$

A left cyclic partition of order $2 p-3$ in $H_{n+1}$ is obtained from the left cyclic partition exhibited in the case $p \geq 5$ by replacing the subsequence $1 S_{2}, 0 S_{2}, 0 S_{3}$, $1 S_{3}, 1 S_{4}, 0 S_{4}, 0 S_{5}, 1 S_{5}$ by $1 S_{2}, 0 S_{2} \cup 1 S_{3}, 0 S_{3}, 0 S_{4}, 1 S_{4} \cup 0 S_{5}, 1 S_{5}$.

Lemma 6. $\mathrm{H}_{4}$ admits left cyclic partitions of orders 5 and 7.

Proof.

- A left cyclic partition of order 5 in $H_{4}$ is the following :

$\{0000,1101\},\{0001,1100,0010,1111\},\{0110,1011\},\{0100,0111,1001,1010\}$, $\{0011,0101,1000,1110\}$.

- A left cyclic partition of order 7 in $H_{4}$ is the following :

$\{0000,1101\},\{0001,1100\},\{0011,1110\},\{0010,1111\},\{0110,1011\}$, $\{0100,0111,1001,1010\},\{0101,1000\}$. 
Lemma 7. $H_{n}, n \geq 4$, admits a left cyclic partition of order $2^{n-1}-1$.

Proof. Consider the sequence $\left\{u_{i} ; 0 \leq i \leq 2^{n-1}-1\right\}$, defined by $u_{i}=\operatorname{bin}(i) \oplus$ $\operatorname{bin}(i / 2)$, where $\operatorname{bin}(x)$ is the $n$-position binary representation of the integer $x$, and symbol / denotes integer division. It can be easilly checked that this sequence corresponds to a hamiltonian cycle in $H_{n-1}$. Now, let us denote $v_{i}=u_{i} \oplus 1 \oplus 2^{n-2}$ (i.e. $v_{i}$ is obtained from $u_{i}$ by changing the first and last bits) and $N=2^{n}$. It is also easy to check that $\left\{v_{i} ; 0 \leq i \leq 2^{n-1}-1\right\}$ is a hamiltonian cycle of $H_{n-1}$. Now, observe that $0 u_{i} \oplus 1 v_{i}=2^{n-1} \oplus\left(u_{i} \oplus v_{i}\right)=2^{n-1} \oplus 1 \oplus 2^{n-2}$. Hence, $0 u_{i}$ and $1 v_{i}$ are not neighbors in the hypercube $H_{n}$. On the other hand, $u_{N-4}=100 \ldots 010$, $u_{N-2}=10 \ldots 01, u_{N-1}=10 \ldots 0$ and $v_{0}=u_{0} \oplus 1 \oplus 2^{n-2}=10 \ldots 01=u_{N-2}$. Hence, $0 u_{N-4}, 0 u_{N-1}, 0 u_{N-2}, 1 v_{0}$ is a chain of $H_{n}$. Moreover, $v_{N-4}=0 \ldots 011$, $v_{N-2}=0 \ldots 0=u_{0}$ and $v_{N-1}=0 \ldots 01=u_{1}$. Hence $1 v_{N-4}, 1 v_{N-1}, 1 v_{N-2}, 0 u_{0}$ is a chain of $H_{n}$. Hence, by considering the two chains and the two previous hamiltonian cycles, we see that the partition $\left\{0 u_{0}, 1 v_{0}\right\},\left\{0 u_{N-4}, 1 v_{N-4}\right\}$, $\left\{0 u_{N-3}, 0 u_{N-1}, 1 v_{N-3}, 1 v_{N-1}\right\},\left\{0 u_{N-2}, 1 v_{N-2}\right\}$ is a left cyclic partition.

Proposition 3. $H_{n}, n \geq 4$, admits left cyclic partitions of all odd orders from 5 to $2^{n-1}-1$.

Proof. We proceed by induction. For $n=4$ the result follows from lemma 6 . Assuming that the result holds for $n \geq 4$, let us consider an $(n+1)$-cube together with an odd integer $p \in\left[5,2^{n}-1\right]$.

- Case $1: 5 \leq p \leq 2^{n-1}-1$. The result follows from the induction hypothesis and lemma 4 .

- Case $2: 2^{n-1}-1<p<2^{n}-1$. There is an odd integer $q, 7<q<2^{n-1}-1$, such that $p=2 q-1$ or $p=2 q-3$. The result follows from the induction hypothesis and lemma 5 .

- Case $3: p=2^{n}-1$. The result follows from lemma 7

Theorem 1. The cycles generated by the parallel chip firing game associated with $n$-cube orientations, $n \geq 4$, are of even lengths from 2 to $2^{n}$, and of odd lengths different from 3 and ranging from 1 to $2^{n-1}-1$.

\section{Conclusion}

In this paper, we have given, in the particular case of parallel evolutions, a range of possible periods which can be generated by the chip firing game associated with $n$-cube orientations. The question of the existence of cycles with length greater than $2^{n}$ remains to be clarified. We suggest, for the case of $n$-cubes, to consider a recurrent approach based on the sub-evolutions induced in every face of the $n$-cube by a block sequential evolution.

\section{References}

1. M. A. Kiwi, R. Ndoundam, M. Tchuente and E. Goles No polynomial bound for the period of the parallel chip firing game on graphs, Theoretical Computer Science, 136, pp. 527-532, 1994. 